\title{
Détermination de l'efficacité biologique relative des neutrons de fission en fluence mixte neutron-gamma pour la survie globale des cellules germinales du rat mâle
}

\author{
G. LEMAIRE, J. MAAS et G. GRILLON (*) \\ (Manuscrit reçu le 15 juin 1981)
}

\begin{abstract}
RÉSUMÉ
A partir de la survie globale in vivo des cellules germinales du rat mâle après irradiation, retenue comme critère biologique, les auteurs déterminent les valeurs numériques de l'EBR en fluence mixte neutron gamma pour différents rapports de dose neutron sur gamma. Ces valeurs avoisinent 10 pour quelques décigray (1-3 dGy).

II ressort de cette étude expérimentale l'absence d'interaction entre les effets biologiques des rayonnements gamma et neutronique : la survie globale des cellules germinales en champ mixte est égale au produit des survies respectivement obtenues avec les gamma et avec les neutrons. II en résulte que I'EBR à prendre en considération en champ mixte est celui correspondant à la composante neutronique à la dose totale.

Ces résultats amènent les auteurs à penser que lors d'irradiations en champ mixte de grands mammifères, le gradient de dose neutronique qui s'établit à l'intérieur de l'organisme pourrait être compensé par les valeurs numériques élevées des EBR aux faibles doses. L'essai de vérification de cette hypothèse est actuellement en cours.
\end{abstract}

\section{ABSTRACT}

Taking total germinal cell survival after irradiation in the rat as a biological criterion, RBE's coefficients were determined in mixed fields (neutron + gamma) for various ratios of neutron/gamma doses. Numerical values can reach 10 for low dose levels (1-3 dGy).

It can be concluded that the biological effects of gamma rays and neutrons seem to be independent: RBE's values do not depend upon the neutron/gamma ratio ; thus, in mixed fields, they correspond to the neutron dose only.

These experimental results show that in mixed field irradiations of large animals, the high RBE values might make up for the gradient of neutron dose which exists inside the body. Experiments are being made to check this assumption.

(*) Commissariat à l'énergie atomique IPSN, Département de protection. Groupe mixte de recherche, BP 6, 92260 Fontenay-aux-Roses. 


\section{INTRODUCTION}

Les champs neutroniques obtenus par les différents moyens d'irradiation actuellement disponibles ne sont jamais purs; ils comportent toujours une proportion variable de rayonnements gamma. Les irradiations sont donc toujours réalisées en champ mixte. Dans ces conditions expérimentales, la connaissance du "poids relatif" de la dose délivrée par chaque type de rayonnement revêt un intérêt pratique, ne serait-ce que pour les applications radiothérapeutiques de tels faisceaux.

En prenant pour référence l'éfficacité biologique des rayonnements gamma, celle des neutrons est représentée par le rapport des doses gamma sur neutron qui provoquent le mëme effet biologique, "toutes choses étant égales par ailleurs".

Cette notion d'EBR (rapport des doses de deux rayonnements, dont un de référence, conduisant au même effet biologique) qui traduit, en fait, notre ignorance des mécanismes initiaux mis en jeu, est fondamentale en radioprotection où les facteurs de qualité ne sont entre autres que le résultat global de l'intégration des EBR relatifs aux différents critères retenus.

Pour déterminer l'EBR des neutrons en fluence mixte, il faut disposer d'une source d'irradiation où l'on peut faire varier les composantes gamma et neutrons de la dose totale délivrée, établir les relations dose-effet pour les différents rapports de doses neutron-gamma et comparer ces relations à celle obtenue en irradiation gamma pure.

C'est dans cet esprit que nous avons entrepris une série d'irradiations auprès du réacteur Silène où la composante gamma peut être réduite en disposant autour de "son cœur" des écrans de plomb sans trop déformer, par ailleurs, le spectre des neutrons émis et auprès du réacteur Harmonie qui en configuration bloc acier peut être considéré comme une source de neutrons purs à $4 \%$ près.

Dans cette étude, le critère biologique retenu dans la définition de l'EBR est la "survie globale" in vivo des cellules germinales du rat mâle déduite de l'involution pondérale $\left(^{\star}\right)$ de leurs testicules, le rayonnement de référence étant les rayonnements gamma du cobalt -60 .

\section{METHODES EXPERIMENTALES}

\section{II.1. Animaux}

Les expérimentations ont été effectuées sur des rats mâles Sprague Dawley descendants (souche OFA d'IFFA CREDO) âgés de 3 mois environ et pesant près de $360 \mathrm{~g}$ le jour de leur irradiation. L'importance numérique des lots utilisés ( 40 rats chacun) nous a permis de rendre ceux-ci très homogènes par une répartition selon le poids corporel réalisée dès l'instant où la courbe de croissance reprend sa forme régulière après l'arrivée des animaux au laboratoire. Les rats d'un même lot sont mis en cagettes cylindriques individuelles en aluminium réparties sur une surface isodose.

(*) Involution: perte de poids ou de volume. 
A partir du $8^{e}$ jour qui suit l'irradiation, ils sont prélevés par cage de 4 pour éviter tout "effet cage", selon un rythme bihebdomadaire. Le poids corporel et celui des gonades débarrassées de leurs enveloppes extérieures sont mesurés chez les rats irradiés et chez les rats témoins; les testicules sont ensuite fixés en vue des examens histologiques.

\section{II.2. Sources d'irradiation}

\section{II.2.1. Réacteur Silène (CEN de Valduc)}

Les neutrons sont produits par la divergence contrôlée d'une solution de nitrate d'uranyle enrichi à $93 \%$ en uranium 235. La mise en place d'un écran de plomb autour du cœur permet de réduire considérablement la dose gamma : le rapport dose gamma/dose neutron passe de 1,2 à 0,17 . La figure 1 représente la fluence neutronique relative en fonction de l'énergie des neutrons (sans écran de plomb). L'énergie moyenne est de l'ordre de $1 \mathrm{MeV}$.

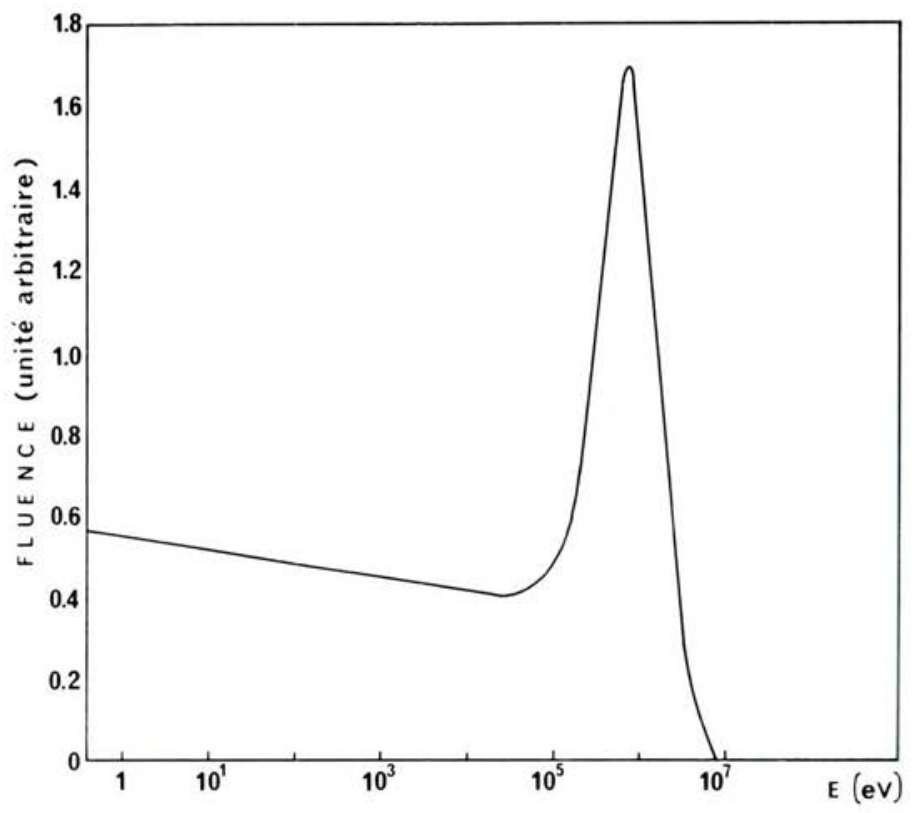

Fig. 1. - Spectre des neutrons émis par le réacteur Silène.

La dosimétrie a été réalisée par le laboratoire de dosimétrie sanitaire du Département de protection (CEN de Fontenay-aux-Roses) en appliquant la méthode des doubles chambres [10]:

- la dose totale $(n+\gamma)$ est mesurée avec une chambre d'ionisation à paroi et gaz équivalent aux tissus (Shonka) ; 
- la dose gamma est mesurée avec une chambre d'ionisation en aluminium balayée par de l'argon, dont la sensibilité aux neutrons est déterminée à l'aide d'un compteur Geiger-Müller peu sensible aux neutrons.

Les doses neutron et gamma sont calculées à l'aide d'un système à double équation [2].

La dose entre rats fluctue au maximum de $10 \%$. En raison du très grand avantage présenté par l'irradiation unique d'un lot important d'animaux, nous avons tout de même considéré que les 40 rats de chaque lot recevaient la même dose. Eu égard à la taille relativement réduite des testicules du rat et à leur position externe, nous avons admis que la dose absorbée par les gonades avait même valeur que le kerma.

Le débit de kerma total a été de 0,02 Gy.min¹.

\subsubsection{Reacteur Harmonie (CEN de Cadarache)}

C'est un réacteur à neutrons rapides de faible puissance, destiné à fournir des neutrons de fission.

La figure 2 représente le spectre des neutrons émis par ce réacteur dans la configuration bloc acier avec laquelle nous avons travaillé. Compte tenu des dimensions importantes du hangar qui surplombe Harmonie, on peut considérer négligeable la fraction de dose due aux rayonnements diffusés. La gamme énergétique des neutrons émis s'étend de $10 \mathrm{keV}$ à $1,5 \mathrm{MeV}$. La contamination gamma du faisceau est inférieure à $4 \%$. Les animaux ont été irradiés quatre par quatre, leur espacement étant suffisant pour que la présence de l'un d'entre eux n'altéra point la fluence atteignant les autres. La valeur numérique du débit de kerma total dans l'air a toujours été fixée à 0,15 Gy. minn-1 $^{-1}$.

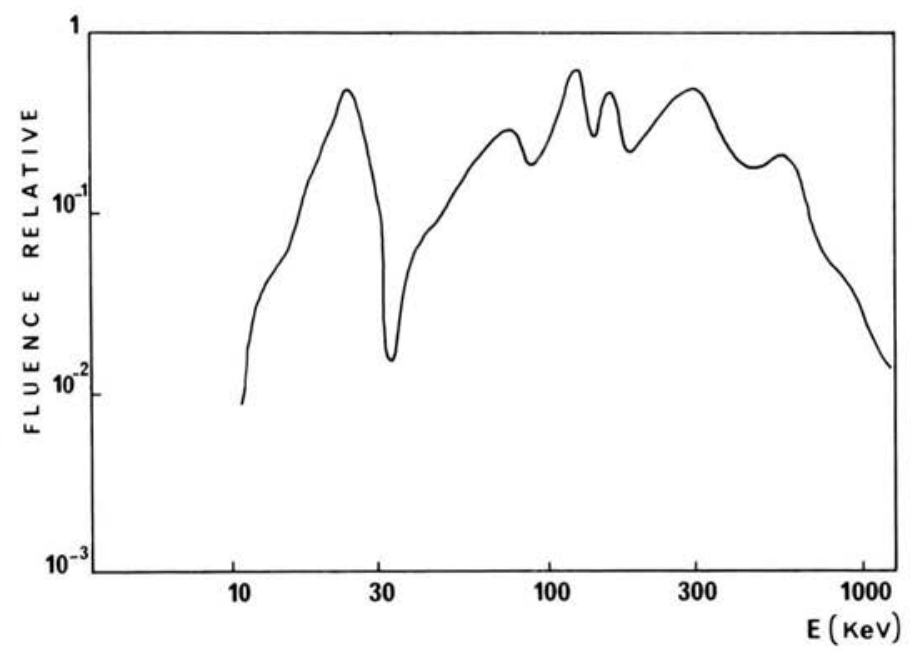

Fig. 2. - Spectre des neutrons émis par le réacteur Harmonie. 


\section{RESULTATS EXPERIMENTAUX - DISCUSSION}

\section{III.1. Involution pondérale des testicules du rat après irradiation aiguë}

A partir d'une certaine dose absorbée dont la valeur diffère avec la nature du rayonnement, l'irradiation aiguë des testicules du rat provoque une modification de leurs structures qui se traduit au plan macroscopique par la diminution de leur volume et de leur poids [4-6]. Ce phénomène a été mis en évidence pour la première fois par Eschenbrenner [1].

La perte de substance commence à être décelable quelques heures après l'irradiation et s'accentue progressivement pour atteindre un maximum à partir du $28^{e}$ jour. Cette involution est linéaire comme le prouvent les tests de linéarité et la valeur absolue de la pente croît avec la dose de rayonnement (fig. 3).
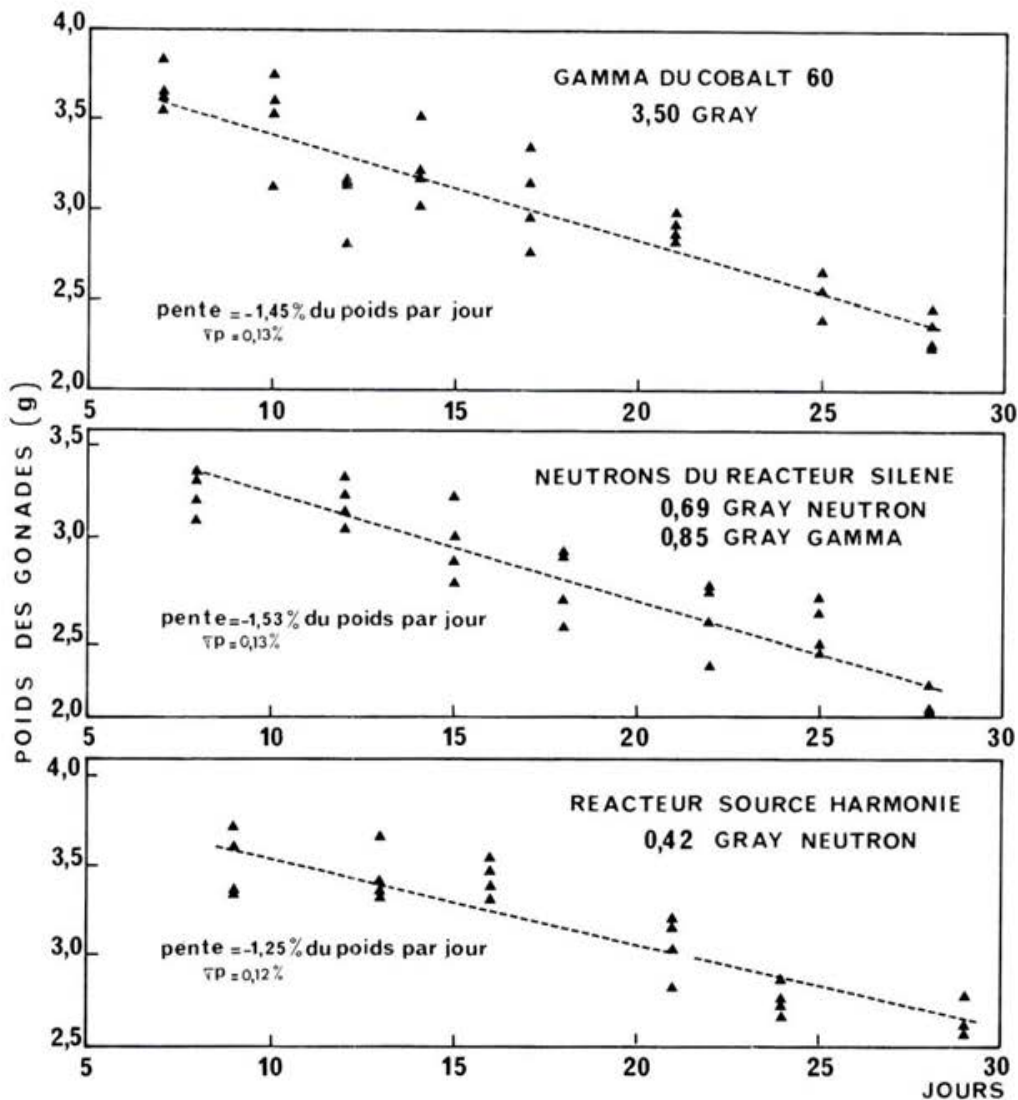

Fig. 3. - Involution pondérale des testicules du rat après irradiation aiguë. 
L'équation représentative de l'involution du poids $P$ des testicules avec le temps est donc:

$$
P=P_{0}+A \cdot t
$$

soit $P=P_{\circ}\left(1+\frac{p . t}{100}\right)$ avec $p=\frac{A}{P_{0}} \times 100$

Les valeurs de $P_{0}$ oscillent entre 3,5 et $4 \mathrm{~g}$ ce qui est très proche du poids des gonades $(3,6 \mathrm{~g})$ des rats témoins de même poids corporel le jour de l'irradiation.

\section{III.2. Survie globale des cellules germinales mâles}

La survie globale $\mathrm{S}$ des cellules germinales mâles est définie par la proportion de toutes les cellules germinales des deux testicules encore présentes après l'irradiation de ceux-ci. Le poids des cellules étant proportionnel à leur nombre, elle est représentée par le rapport du poids $\mathrm{Pg}$ du tissu germinal des irradiés au poids $\mathrm{Pg}_{\circ}$ de ce tissu chez les témoins.

$\mathrm{Pg}$ et $\mathrm{Pg}$ 。 s'obtiennent en soustrayant du poids des gonades des irradiés $(P)$ ou des témoins $\left(P_{0}\right)$ le poids $W$ de la composante non germinale des testicules peu radiosensible (fibrocytes, cellules de Sertoli et de Leydig). Nous avons déterminé la valeur de $\mathrm{W}$ chez des témoins par analyse manuelle ou semi-automatique des surfaces occupées sur les coupes histologiques respectivement par les parties germinales et somatiques de la gonade du rat mâle ; elle représente $37,4 \pm 0,8 \%$ de $P_{o}$. Elle garde cette valeur chez les irradiés car nous avons vérifié que les cellules somatiques ne sont pas sensibles aux rayonnements gamma et neutronique dans la gamme des doses analysées (dose gamma inférieure à 5 Gy, doses gamma et neutron concomitantes respectivement inférieures ou égales à 2,78 et 2,25 Gy).

Nous avons montré [7] que la survie globale des cellules germinales du rat mâle peut être déduite du poids des gonades après irradiation quand

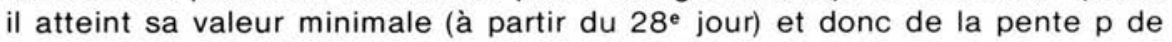
l'involution pondérale puisque celle-ci est linéaire.

A noter que la pente traduit la cinétique du phénomène et est donc plus caractéristique que le poids mesuré à un instant donné.

En se plaçant au $28^{\mathrm{e}}$ jour, on peut donc écrire :

$S=\frac{P_{g}}{P_{g \circ}}=\frac{P-W}{P_{0}-W}=\frac{P_{0}(1+0,28 p)-0,374 P_{0}}{P_{0}-0,374 P_{0}}=1+0,447 p$

$p$ étant exprimé en $\%$. jour ${ }^{-1}$. 
Les résultats sont illustrés, en fonction de la dose absorbée, par la figure 4 qui comporte quatre courbes:

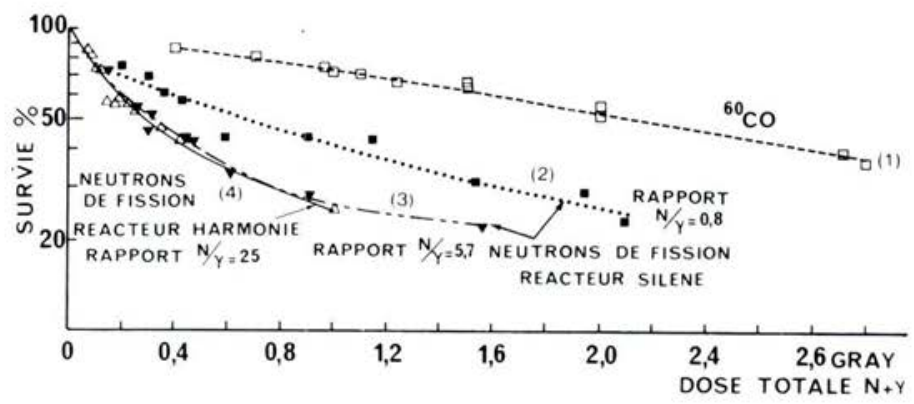

Fig. 4. - Irradiation aiguë : survie germinale globale (rat mâle).

La courbe 1 est la courbe de survie en irradiation gamma pure. En admettant un modèle de Kellerer et Rossi [3] son équation représentative est de la forme:

$$
\mathrm{S}=\mathrm{e} \cdot \alpha^{\mathrm{D}} \mathrm{e} \cdot \beta \mathrm{D}^{2} \text { avec } \alpha=28,2 \cdot 10^{-2} \mathrm{~Gy}^{-1} \text { et } \beta=8,3 \cdot 10^{-3} \mathrm{~Gy}^{-2}
$$

Les courbes 2 et 3 sont les courbes de survie en fluence mixte neutron gamma du réacteur Silène, en fonction de la dose absorbée totale et pour les deux rapports des doses neutron et gamma (rapport inférieur à 1 et supérieur à 1 respectivement). Elles sont obtenues par ajustement au sens des moindres carrés.

La courbe 4 est la courbe de survie obtenue avec le faisceau neutronique presque pur du réacteur Harmonie [8].

\section{DISCUSSION}

Les courbes de survie 2 à 4 , obtenues en fluence mixte neutron gamma, sont à épaulement inverse (concavité tournée vers le haut). L'épaulement inverse traduit généralement une hétérogénéité cellulaire, en l'occurrence l'existence de populations de cellules à radiosensibilité différente.

En ce qui concerne la chaîne spermatogénétique, il est bien connu que les cellules initiatrices (spermatogonies $A$, intermédiaires et $B$ ) sont sensibles à quelques dixièmes de Gray tandis que les cellules suivantes (spermatocytes I et II, spermatides spermatozoïdes) résistent à quelques Gray.

En irradiation gamma pure, la courbe de survie 1 ne représente pas cet épaulement inverse, du moins jusqu'à la dose de $3 \mathrm{~Gy}$, ce qui signifie que les cellules initiatrices se comportent comme faisant partie d'une seule population ou encore que la radiosensibilité de chacun de leurs stades est du même ordre de grandeur. 
En fluence mixte, l'hypothèse de l'existence de populations cellulaires à radiosensibilité différente ne peut pas être retenue pour deux raisons. D'une part, les doses délivrées n'étant, en général, que de quelques dixièmes de Gray de neutrons et de gamma, seules les cellules les plus radiosensibles (spermatogonies $A$, intermédiaires et $B$ ) sont concernées. Ceci est confirmé par l'histologie : l'irradiation gamma pure ou gamma + neutron ne modifie ni les proportions des types d'associations cellulaires présentées par les tubules séminifères ni les proportions des cellules dérivées des spermatogonies $A$. D'autre part, la variation des proportions des populations radiosensible et radiorésistante en fonction de la valeur du rapport dose neutron / dose gamma, signifiée par les différences de pente des parties initiales ou finales des courbes 2 à 4 , ne se trouve pas vérifiée au plan histologique, la composition cellulaire des tubules séminifères restant inchangée quel que soit le rapport des doses.

II semble alors qu'en fluence mixte l'épaulement inverse des courbes de survie provienne de la non équivalence des doses neutron et gamma et donc de l'impossibilité de les sommer pour établir ces courbes.

De fait, l'examen des courbes 1 à 4 aboutit à la constatation suivante : pour une même dose absorbée totale la survie est d'autant plus faible que la fraction de dose due aux neutrons est plus grande ou, ce qui revient au même, pour obtenir une survie donnée, la dose absorbée totale à délivrer est d'autant plus réduite que l'irradiation comprend une plus grande proportion de neutrons.

On ne peut pas attribuer le même "poids", la même efficacité biologique, à chaque type de rayonnement.

Si nous admettons que les mécanismes d'action des deux rayonnements sont indépendants comme le suggère l'analyse histologique qui montre une même évolution du tissu germinal après irradiation gamma pure ou neutron + gamma, la survie totale en fluence mixte est alors le produit des survies aux gamma et aux neutrons:

$\mathrm{S}_{\mathrm{dt}}=\mathrm{S}_{\mathrm{dn}} \cdot \mathrm{S}_{\mathrm{d} \gamma}$.

$d_{n}$ et $d \gamma$ étant les doses de neutrons et de gamma $(d t=d n+d \gamma$ ).

$S_{d} \gamma$ est connue à partir de (1). $S_{d n}$ peut donc être calculée. A la dose $d_{n}$ correspond une dose d' $\gamma$ en irradiation gamma pure pour obtenir la même survie $S_{d n}$. L'EBR est alors égal à :

$$
\mathrm{EBR}=\frac{\mathrm{d}^{\prime} \gamma}{\mathrm{d} n}
$$

Aux erreurs expérimentales près, dans la gamme de dose neutron commune explorée $(0,17$ à $1,34 \mathrm{~Gy})$ les valeurs EBR obtenues sont très voisines pour une même dose neutron quand le rapport dose neutron / dose gamma passe de 0,6 à 25 , et diminuent pareillement quand la dose neutron augmente (fig. 5).

Le premier fait montre que l'hypothèse de l'indépendance des actions des rayonnements neutronique et gamma appliquées simultanément est justifiée et donc qu'en champ mixte l'EBR neutron à considérer est celui correspondant à la composante neutronique. 


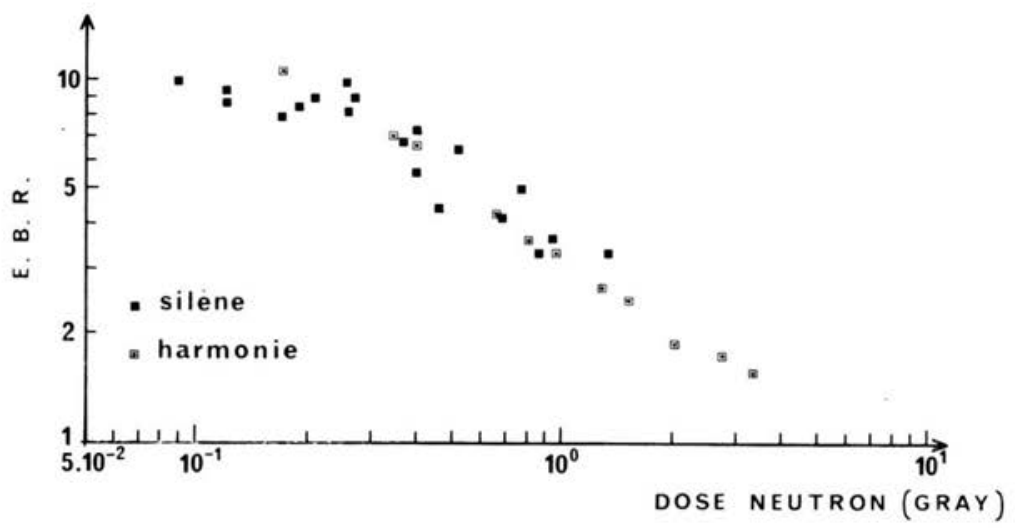

VARIATION DE L'E.B.R. DES NEUTRONS EN FONCTION DE LA DOSE NEUTRONIQUE EN FLUENCE MIXTE $n+r$

Fig. 5. - Irradiation aiguë : survie germinale (rat mâle).

Le deuxième fait peut être précisé à l'aide du calcul des paramètres constants de la droite de régression entre le logarithme de I'EBR et celui de la dose neutron $d_{n}$ :

$\log E B R=A+B . \log d_{n}$

Nous trouvons :

\begin{tabular}{|c|c|c|c|c|c|}
\hline \multirow{3}{*}{ Silène } & Rapport $\mathrm{n} / \boldsymbol{\gamma}$ & $\mathrm{A}$ & $\mathrm{s}^{2}{ }_{\mathrm{A}}$ & $\mathrm{B}$ & $\mathrm{s}^{2} \mathrm{~B}$ \\
\cline { 2 - 6 } & 0,6 à 0,8 & 1,2023 & 0,0072 & $-0,5637$ & 0,0063 \\
Harmonie & 5 à 6 & 1,4281 & 0,0031 & $-0,5523$ & 0,0029 \\
\hline & 25 & 1,1793 & 0,0006 & $-0,7013$ & 0,0007 \\
\hline
\end{tabular}

Au risque $5 \%$, la pente $B$ n'est pas significativement différente pour Silène quand le rapport $n / \gamma$ passe de 0,6 à 6 , mais l'est entre Silène et Harmonie, ce qui peut provenir de la non équivalence complète des spectres en énergie des neutrons émis par les deux réacteurs et des débits de kerma utilisés.

Par ailleurs, les valeurs de B sont proches de $-0,5$ pour la gamme de doses neutron considérée et les valeurs de l'EBR semblent tendre vers une limite pour les doses neutron inférieures à 17 cGy (fig. 5). Ceci est conforme à la théorie de Rossi sur la survie cellulaire [11].

Cette augmentation de la valeur de l'EBR des neutrons avec la diminution de la dose neutronique entraîne, pour la prévision de l'effet global, la nécessité de connaître la distribution dans l'organisme de la dose neutron si celle-ci est hétérogène, ce qui est le cas des grands animaux puisqu'un fort gradient de dose neutron s'y établit en irradiation unilatérale. 


\section{CONCLUSION}

Pour le critère "survie globale" des cellules germinales du rat mâle déduite de l'involution pondérale des gonades après irradiation, les expérimentations que nous avons réalisées en fluence mixte neutron-gamma selon différents rapports de doses neutron/gamma, permettent la détermination des coefficients EBR à appliquer au rayonnement le plus ionisant, en l'occurrence, les neutrons. En fluence mixte neutron-gamma les coefficients EBR des neutrons ne dépendent pas du rapport des doses neutron/gamma concomitantes. Ils ne sont fonction que de la fraction de dose totale, due aux neutrons (leur valeur varie avec la dose neutronique).

Les conséquences de ces résultats expérimentaux obtenus in vivo aux niveaux cellulaire et tissulaire sont multiples:

- II n'y aurait pas interaction entre les effets biologiques des rayonnements neutroniques et gamma. En particulier, il n'y aurait ni synergie. La survie globale des cellules germinales du rat mâle en fluence mixte apparaît ainsi égale au produit des survies en ambiance neutron pure et en ambiance gamma pure, chacune de celles-ci étant indépendante de l'autre.

- En fluence mixte neutron-gamma, la dose absorbée totale ne suffit, pas, à elle seule, pour prédire l'effet biologique global. En radioprotection, lorsque des rayonnements de nature différente sont impliqués en même temps, il est nécessaire de bien connaître les diverses composantes de la dose absorbée totale pour évaluer les effets ou les risques globaux.

- Pour les grands animaux irradiés en fluence mixte neutron-gamma, on peut prévoir que vis-à-vis de l'effet biologique, l'affaiblissement de la dose neutron avec la profondeur sera en partie compensé par l'augmentation de la valeur de l'EBR des neutrons quand la dose neutron décroît (aux faibles doses, l'EBR des neutrons peut dépasser 10).

II est donc important de préciser ces différents points en poursuivant cette étude grâce à l'emploi d'un dispositif d'irradiation délivrant simultanément deux rayonnements à transfert d'énergie linéique différent et permettant de maîtriser la qualité spectrale du faisceau mixte comme de varier à volonté le rapport des doses délivrées par ces deux rayonnements. Ce dispositif pourrait comprendre un générateur de rayons $X$ durs et un accélérateur de particules. C'est ce que nous essaierons de réaliser dans les années à venir.

\section{BIBLIOGRAPHIE}

[1] ESCHENBRENNER A.B., MILLER E., LORENZ E. - Quantitative histological analysis of the effect of chronic whole body irradiation with gamma - rays on the spermatogenic elements and interstitial tissue of the testis of mice. J. Nat. Cancer Inst., $1948,9,133-147$.

[2] ICRU. - Neutron dosimetry for biology and medicine, ICRU report 26. Washington : ICRU, 1977. 
[3] KELLERER A.M., ROSSI H.M. - The theory of dual radiation action. Curr. Top. Radiat. Res., 1972, 8, 85-158.

[4] KOHN H.I., KALLMAN R.F. - Testis weight loss, a quantitative measure of $X-$ ray injury in the mouse, hamster and rat. Brit. J. Radiol., 1954, 27, 586-591.

[5] LEGEAY G., GUENET J.L., TARDY - JOUBERT P. - Efficacité biologique relative des protons de $3 \mathrm{GeV}$ sur la gonade du rat mâle. In : $2^{\circ}$ Congrès international de radioprotection autour des accélérateurs et dans l'espace, Genève, 26-30 avril 1971. Genève: CERN, 1971, vol. 1, 84-101.

[6] LEMAIRE G., GRILLON G. - Involution des gonades du rat après irradiation aux rayonnements gamma du cobalt -60 et aux neutrons. Note CEA - N - 1678, 1973.

[7] LEMAIRE G. - Radiobiologie quantitative : méthode d'étude in vivo de cellules germinales souches sous rayonnement gamma et neutronique, modèles descriptifs. Thèse Doctorat d'Etat, Université René Descartes, Paris V, 1976.

[8] LEMAIRE G., GRILLON G. - Détermination des coefficients d'efficacité biologique relative (EBR) des neutrons. Son importance dans l'évolution des dommages biologiques. Applications militaires. Note ETCA - 76 - R - 0,74, 1977.

[9] LEMAIRE G., GRILLON G. - Testis weight loss as a quantitative measure of spermatogonia A survival in the rat "in vivo" after gamma-ray or neutron irradiation. Communication au $13^{\circ}$ congrès annuel de la Société européenne de radiobiologie, Liège, $17 \cdot 19$ octobre 1977.

[10] RICOURT A., NGUYEN V.D., MEDIONI R., PERRIER J.C. - Campagne internationale d'intercomparaison sur la dosimétrie neutronique faite au TNO et au GSF (octobre et novembre 1975). Rapport CEA - R - 4793, 1976.

[11] ROSSI H.H. - The effects of small doses of ionizing radiation. Phys. Med. Biol., $1970,15,255-262$. 